\title{
Erratum to: FDG-PET/ceCT is useful to predict recurrence of Pseudomyxoma peritonei
}

\author{
Julien Dubreuil $^{1,2}$ - Francesco Giammarile ${ }^{3,4}$ - Pascal Rousset ${ }^{2,5}$ - Naoual Bakrin ${ }^{6}$. \\ Guillaume Passot $^{7}$ - Sylvie Isaac ${ }^{8}$ - Olivier Glehen ${ }^{2,7,9}$ - Andrea Skanjeti ${ }^{1,2}$
}

Published online: 5 April 2016

(C) Springer-Verlag Berlin Heidelberg 2016

Erratum to: European Journal of Nuclear Medicine and Molecular Imaging

DOI 10.1007/s00259-016-3347-z

The original version on this paper contained an error.

The names of all authors were displayed incorrectly.

The names and surnames of the authors are inversed.

The author names should appear as "name, surname":

Julien Dubreuil, Francesco Giammarile, Pascal Rousset, Naoual Bakrin, Guillaume Passot, Sylvie Isaac, Olivier Glehen, Andrea Skanjeti

The online version of the original article can be found at http://dx.doi.org/ 10.1007/s00259-016-3347-z.

Julien Dubreuil

julien.dubreuil@chu-lyon.fr

1 Nuclear Medicine and PET Unit, Centre Hospitalier Lyon-Sud, Hospices Civils de Lyon, 165 Chemin du Grand Revoyet, 69495 Pierre-Bénite, France

2 Equipe Mixte de Recherche 3738, Université Claude Bernard Lyon 1, Lyon, France

3 Nuclear Medicine Department, Groupement Hospitalier Est, Hospices Civils de Lyon 59, boulevard Pinel, 69677 Bron, France

4 Biophysique - Faculté Charles Mérieux Lyon, Lyon, France
5 Radiology Department, Centre Hospitalier Lyon-Sud, Hospices Civils de Lyon, 165 Chemin du Grand Revoyet, 69495 Pierre-Bénite, France

6 Gynaecology Department, Groupement Hospitalier Est, Hospices Civils de Lyon, 59, boulevard Pinel, 69677 Bron, France

7 General and oncologic surgery Department, Centre Hospitalier Lyon-Sud, Hospices Civils de Lyon, 165 Chemin du Grand Revoyet, 69495 Pierre-Bénite, France

8 Pathology Department, Centre Hospitalier Lyon-Sud, Hospices Civils de Lyon, 165 Chemin du Grand Revoyet, 69495 Pierre-Bénite, France

9 Lyon 1 University, 43 Boulevard du 11 Novembre 1918, 69100 Villeurbanne, France 